\title{
The influence of star clusters on galactic disks: new insights in star-formation in galaxies
}

\author{
Pavel Kroupa \\ Argelander-Institut für Astronomie, University of Bonn, Auf dem Hügel 71, \\ D-53121 Bonn, Germany \\ email: pavel@astro.uni-bonn.de
}

\begin{abstract}
Stars form in embedded star clusters which play a key role in determining the properties of a galaxy's stellar population. A large fraction of newly born massive stars are shot out from dynamically unstable embedded-cluster cores spreading them to large distances before they explode. Embedded clusters blow out their gas once the feedback energy from the new stellar population overcomes its binding energy, leading to cluster expansion and in many cases dissolution into the galaxy. Galactic disks may be thickened by such processes, and some thick disks may be the result of an early epoch of vigorous star-formation. Binary stellar systems are disrupted in clusters leading to a lower fraction of binaries in the field, while long-lived clusters harden degenerate-stellar binaries such that the SNIa rate may increase by orders of magnitude in those galaxies that were able to form long-lived clusters. The stellar initial mass function of the whole galaxy must be computed by adding the IMFs in the individual clusters. The resulting integrated galactic initial mass function (IGIMF) is top-light for SFRs $<10 M_{\odot} / \mathrm{yr}$, and its slope and, more importantly, its upper stellar mass limit depend on the star-formation rate (SFR), explaining naturally the mass-metallicity relation of galaxies. Based on the IGIMF theory, the re-calibrated $\mathrm{H} \alpha$-luminosity-SFR relation implies dwarf irregular galaxies to have the same gas-depletion time-scale as major disk galaxies, implying a major change of our concept of dwarf-galaxy evolution. A galaxy transforms about 0.3 per cent of its neutral gas mass every $10 \mathrm{Myr}$ into stars. The IGIMF-theory also naturally leads to the observed radial $\mathrm{H} \alpha$ cutoff in disk galaxies without a radial star-formation cutoff. It emerges that the thorough understanding of the physics and distribution of star clusters may be leading to a major paradigm shift in our understanding of galaxy evolution.
\end{abstract}

Keywords. stellar dynamics, gravitation, methods: n-body simulations, binaries: general, stars: formation, stars: luminosity function, mass function, Galaxy: disk, galaxies: star clusters, galaxies: kinematics and dynamics

\section{Introduction}

Observations of star-formation in the solar vicinity suggest that the majority of stars form in embedded clusters of which only a small fraction survive to become open clusters (Lada \& Lada 2003). A similar finding has emerged for extragalactic systems (Larsen 2002a, Larsen 2002b). And in those galaxies where the star-formation rate is high such that very massive young clusters appear, Larsen(2002b) and Larsen(2004b) argue that there is also no physically meaningful distinction between a "globular-cluster" starformation mode and a "galactic-disk" star-formation mode; a continuum of young-cluster masses is evident. Observations of the embedded cluster mass function (ECMF) show it to be a power-law, with $\beta \approx 2$ (the Salpeter index $\beta=2.35$ is suggested by some studies, Larsen 2002a,Weidner et al. 2004), so that the physical processes related to star clusters that affect galaxies on a global scale can be calculated by integrating over the ECMF. 
The problem of clustered star formation in the context of galaxies can be split into two parts: on the one hand, the physical processes of cluster birth and stellar dynamics within the environment posed by a host galaxy need to be understood, and on the other how these propagate through to galaxy scales requires illumination.

In the following a few particular problems are addressed in turn, rather than providing a comprehensive review of star clusters in galactic disks. From this compilation of problems it can be concluded that a rather remarkable amount of galactic astrophysics follows from relatively simple ideas. The notation used here follows that applied in the Cambody lectures by $\operatorname{Kroupa}(2008)$.

\section{Cluster cores as OB-star ejection engines}

It is still not quite settled whether star clusters form mass segregated, but if not then the time-scale for the massive stars $\left(m>5 M_{\odot}\right)$ to sink towards their centers can be roughly estimated from the equipartition time-scale, $t_{\mathrm{ms}} \approx\left(m_{\mathrm{av}} / m_{\mathrm{massive}}\right) t_{\mathrm{relax}}$, where $m_{\text {massive }}, m_{\text {av }}$ are the masses of massive and average stars, respectively, and $t_{\text {relax }}$ is the two-body relaxation time. For embedded clusters $t_{\mathrm{ms}}$ can be very short, of the order of 0.1 Myr (e.g. the Orion Nebula Cluster, ONC), and so determining whether mass segregation is established by birth is a very hard observational problem.

Irrespective of whether the massive stars from in the cluster centre or not, once they are there they form a dynamically unstable cluster core which is depleted in low-mass stars that have been pushed out of the core region. The core decays by ejecting massive stars on a time-scale $t_{\text {decay }} \approx N_{\mathrm{m}} \times t_{\text {core,cross }}$, where $N_{\mathrm{m}}$ is the number of massive stars in the core and $t_{\text {core,cross }}$ is the core-crossing time. The core-crossing time, $t_{\text {core,cross }}=2 r_{\text {core }} / \sigma_{\text {core }}$, can be estimated from the core radius, $r_{\text {core }}$, and the velocity dispersion of the massive stars in the core, $\sigma_{\text {core }}$, whereby care must be taken to remove binary-star motions. For clusters such as the ONC, $t_{\text {decay }}$ can be $0.01-0.1 \mathrm{Myr}$, which again is much shorter than its age (about $1 \mathrm{Myr}$ ). This suggests that the ONC may have already shot out perhaps 70 per cent of its massive stellar content (Pflamm-Altenburg \& Kroupa 2006). This is consistent with the large observed fraction of runaway massive stars, which has been used by Clarke \& Pringle(1992) to infer the initial dynamical configuration of massive stars as being small groups of binary-rich massive stars without the presence of many low-mass stars.

Typical ejection velocities are $5-100 \mathrm{~km} / \mathrm{s}$, and so a large fraction of massive stars explode $15 \mathrm{pc}$ to $4 \mathrm{kpc}$ away from their birth site, assuming the first and last SN occur 3 and 40 Myr after birth, respectively. A recent study of the distribution of massive stars is available by Schilbach \& Roeser(2008), who find that 91 per cent of their sample stars can be traced to an origin in young clusters. Studying bow-shocks produced by OB stars traveling through the interstellar medium, Gvaramadze \& Bomans(2008) "report the discovery of three bow shocks produced by O-type stars ejected from the open cluster NGC 6611 (M16). One of the bow shocks is associated with the O9.5Iab star HD165319, which was suggested as one of the best examples for isolated Galactic high-mass star formation".

The dynamical fact that massive stars are shot out from unstable cluster cores has barely been incorporated in galaxy evolution models, but is likely to have some important effects. In particular, the existence or non-existence of isolated O-star formation would be an important test of star-formation theories, with important implications for the existence of the $m_{\max }-M_{\text {ecl }}$ relation that enters critically into the IGIMF theory of Section 6. 


\section{Binary systems}

High-resolution observations of star-forming regions have been demonstrating that the fraction of binary systems is very high such that most if not all stars form as binaries (Duchene 1999, Kouwenhoven et al. 2007, Goodwin et al. 2007). Star-formation mostly in triple or quadruple systems is ruled out because their dynamical decay time is far shorter than the age of the observed stellar populations (typically older than $1 \mathrm{Myr}$ ): decayed higher-order multiple systems would add too many single stars to the population such that the resulting binary fraction would be too low in comparison to the observed values (Goodwin \& Kroupa 2005). This sets important boundary conditions for the starformation process, but also for star-cluster evolution models.

Numerical models of star-clusters must therefore begin with a binary fraction near unity (Kroupa, Aarseth \& Hurley 2001, de la Fuente Marcos 1997, Ivanova et al. 2005) to be realistic. The evolution of a cluster then assumes a fascinating wealth of new dynamical detail as the binary population changes through the disruption of soft binaries and hardening of hard binaries (Giersz \& Spurzem 2003, Davies et al. 2006, Heggie et al. 2006, Trenti et al. 2007, Portegies et al. 2007), and the cluster responds by being heated to expand (Meylan \& Heggie 1997), but brief cluster cooling through early binary disruption has also been observed (Kroupa, Petr \& McCaughrean 1999).

For galactic disks the main effects of initially binary-rich clusters are as follows:

- The binary fraction decreases: one rather remarkable result is that the observed high binary fraction among late-type stars in star-forming regions $\left(f_{\text {bin }} \approx 1\right)$ becomes nicely consistent with the observed binary fraction in the Galactic disk $\left(f_{\text {bin }} \approx 0.5\right)$ while at the same time the observed period- and mass-ratio distribution functions are matched as well. A unification between star-forming and disk populations has therewith been achieved (Kroupa 2008).

- The stellar ejection rate from clusters is increased significantly (Kroupa 1998, Küpper et al. 2008) leading to many more stars with velocities larger than $10 \mathrm{~km} / \mathrm{s}$. Binary-rich clusters as stellar accelerators have not been taken into account in galaxy-evolution models.

- The SNIa rate increases: As argued by Shara \& Hurley (2002), the tightening of binary orbits through stellar-dynamical encounters (through sling-shot fly-bys) in longlived clusters leads to degenerate stars becoming tight binaries which is a pathway to supernova type Ia explosions through accretion-induced or merger-induced detonation. Long lived (i.e. massive) open clusters and globular clusters therewith become SNIaproduction engines, and the SNIa rate may go up by a factor of many if not by orders of magnitude in Galaxies that are able to form such clusters. This is possible in galaxies with a sufficiently high star-formation rate (SFR, Larsen 2004b): Only clusters with a life-time longer than about 5 Gyr host an environment dense enough for a sufficiently long time to allow sufficient hardening of the degenerate binaries. For example, clusters of mass $10^{4.5} M_{\odot}$ have such life-times in a Milky-Way type galaxy if they orbit at a distance of about $8 \mathrm{kpc}$ and on circular orbits (Baumgardt et al. 2008). It follows that galaxies with $S F R>0.1-1 M_{\odot} /$ yr are able to produce such clusters (Weidner et al. 2004).

The implications of this type of SFR-dependence of the SNIa rate for chemical-enrichment have not been studied. 


\section{The expulsion of residual gas and thickened galactic disks}

Because only about 30 per cent of the gas within a few pc of a star-cluster-forming cloud core ends up in stars and the remaining gas is expelled, the emerging young cluster expands. If gas-expulsion is explosive, i.e. occurs on a time-scale shorter or comparable to the crossing-time scale of the cluster, then the cluster expands with a velocity comparable to the velocity dispersion of the pre-gas-expulsion embedded cluster. A remnant cluster may survive containing a small fraction of the birth stellar population: we have a young open cluster with an associated expanding OB association (Kroupa et al. 2001). If gas expulsion occurs on a time scale of a few cluster crossing times or longer, then the exposed cluster reacts adiabatically by expanding and looses a much smaller fraction of its birth population (Lada, Margulis \& Dearborn 1984, Baumgardt \& Kroupa 2007).

This naturally leads to a rapid evolution of the cluster mass function (Kroupa \& Boily 2002, Parmentier et al. 2008), such that the exposed-cluster mass function should appear less steep than the embedded-cluster mass function. It may thus be that the embedded-cluster mass function has a Salpeter power-law index $(\beta=2.35)$ while the exposed star-cluster mass function appears with $\beta \approx 2$. Disruptive expulsion of residual gas also implies a natural explanation for the origin of the population II stellar halo as stemming from low-mass clusters that formed in association with todays globular clusters (Baumgardt et al. 2008), given that there is no special mode of globular cluster formation but rather a continuous power-law embedded-cluster mass function, the upper mass limit of which depends on the SFR (Weidner et al. 2004).

Another implication of residual gas expulsion is that galactic disks get thickened because each star-cluster generation has associated with it the unbound young stars lost from the clusters within the first Myr. If the galaxy has a star-formation rate high enough $\left(S F R>\right.$ few $M_{\odot} /$ yr) to allow the occurrence of massive clusters, then the expanding population has a larger velocity dispersion. A galaxy may thus go through episodes of disk-thickening events if it experiences episodes of increased SFR.

A decreasing stellar velocity-dispersion-age relation towards younger stellar ages, as observed for solar-neighbourhood stars, can be accounted for quite naturally if the Milky Way has a somewhat falling SFR over the past 5-10 Gyr, whereby proper account of other disk-heating mechanisms such as scattering of stars on molecular clouds, on spiral density waves and the bar must be taken into account appropriately (Kroupa 2002).

The Milky Way (MW) thick disk may also result from this process: If the very early MW disk went into a star-burst globally such that compact clusters formed throughout the disk with masses ranging up to $10^{5} M_{\odot}$, and if these experienced a rapid phase of residual gas expulsion such that the "popping" clusters lost a large fraction of their stars which expanded into the young MW with a velocity dispersion comparable to the pre-gas-expulsion velocity dispersion of the embedded clusters $(30-50 \mathrm{~km} / \mathrm{s})$, then a thick-disk component with today's thick-dick velocity dispersion can result.

This scenario has received empirical support from the observation of clumpy but straight disks in chain and spiral galaxies from redshifts $z \sim 0.5$ to 5 (Elmegreen \& Elmegreen 2006). Elmegreen \& Elmegreen write that these observations seem "inconsistent with the prevailing model in which thick disks form during the violent impact heating of thin disks and by satellite debris from this mixing." The authors discount the above "popping cluster" scenario on the basis that the observed "clusters" are massive clumps of sizes of a few hundred pc, and because the observed thickness is not likely to be carried through to todays disk galaxies because of adiabatic contraction as a result of thin-disk growth. The Elmegreen \& Elmegreen high-redshift thick disks would thus become thinner as galaxies grow to the present epoch. 
However, the large and massive clumps observed in chain galaxies by Elmegreen \& Elmegreen are most certainly massive star-cluster complexes as observed in the Antennae galaxies, for example (Bastian et al. 2006). Within each star-cluster complex, each individual cluster would "pop" (Fellhauer \& Kroupa 2005), preserving the essence of the above "disk-thickening through popping clusters" theory. If the young clusters are mass-segregated (e.g. Marks et al. 2008), then mostly low-mass stars would be lost with a velocity dispersion of $30-50 \mathrm{~km} / \mathrm{s}$. Such a population would form a thick oblate distribution about the observed young galaxies but would not be detectable. As the thin disks grow to their present masses, the spheroidal component would adiabatically contract and appear as today's thick disk.

Clearly, this scenario for the origin of thick disks does not dependent on satellite mergers and works in any disk galaxy that experienced a major star-burst throughout the disk. The "popping cluster" scenario is attractive because it relies on known physics rather than invoking for example dark-matter substructures that are merely hypothetical at the present time, but it needs more quantitative work in order to formulate predictable quantities such as the parameters of the velocity ellipsoid at different positions in the Galaxy and for different stellar age-groups. Such work is overdue, given that the data accumulated with the GAIA mission will allow very accurate testing of this scenario.

It should be noted that this "popping-cluster" scenario does not exclude some thickdisks to have originated from infalling satellite galaxies. Indeed, infalling satellites are the only viable scenario for producing counter-rotating disks.

\section{The time scale for the birth of a complete star-cluster population}

The stellar IMF describes the distribution of stars that form together in a clusterforming cloud core within a time-scale of a few Myr at most. It is the statistical outcome of the physical processes that act in the core when star formation proceeds.

What about the star-cluster initial mass function, i.e. the mass function of embedded clusters (ECMF)? Star clusters form in regions of a galaxy where the molecular clouds are massive and dense enough, but it is not clear whether an ensemble of freshly formed star clusters can be defined that represent an initial population in the sense of the stellar IMF. In constructing a galaxy, it is useful though to have this tool.

In this context, it is interesting to note that Egusa et al. (2004) find a characteristic time-scale of about $5 \mathrm{Myr}$ for HII regions to appear after the inter-stellar medium assembles in molecular clouds along spiral arms in disk galaxies. Thus, a disk galaxy would be churning out populations of co-eval (within a few Myr) embedded star clusters on this time-scale. Renaud et al. (2008) investigate the regions of cluster formation in interacting galaxies, and find these to be the fully-compressive tidal regions. The time-scale the inter-stellar medium (ISM) spends in these regions, emanating from them as star clusters, is 10 Myr. Thus, even in massively interacting galaxies it seems that the ISM transforms into star clusters on a time-scale of about 10 Myr when the conditions for star formation are given.

An initial star-cluster population, described by an ECMF, $\xi_{\mathrm{ecl}}\left(M_{\mathrm{ecl}}\right)$, forms on some time-scale $\delta t$, and the total mass in stars thus produced is $M_{\mathrm{tot}}=\int_{M_{\mathrm{ecl}, \mathrm{min}}}^{M_{\mathrm{ecl} \text { ax }}} M_{\mathrm{ecl}} \xi_{\mathrm{ecl}}\left(M_{\mathrm{ecl}}\right)$ $d M_{\mathrm{ecl}}=\delta t \times S F R$. Here $M_{\mathrm{ecl}}$ is the stellar mass in the embedded cluster, and the minimum and maximum values are $M_{\mathrm{ecl} \text {,min }} \approx 5 M_{\odot}$ (Taurus-Auriga-type star-formation) and $1=\int_{M_{\mathrm{ecl}, \mathrm{max}}}^{\infty} \xi_{\mathrm{ecl}}\left(M_{\mathrm{ecl}}\right) d M_{\mathrm{ecl}}$ since there is only one most-massive cluster assuming there to be no bound on the physically maximum cluster mass. 
This set of equations relates the current SFR and $M_{\text {ecl,max }}$. Using the Larsen-sample of star clusters younger than about $10 \mathrm{Myr}$ in star-forming galaxies (chosen independently to the above studies and in order to minimise the effects of early cluster dissolution), Weidner et al. (2004) (WKL) fit the theoretical $M_{\text {ecl,max }}$ vs $S F R$ relation to the observational data. The best-fitting relation has a power-law ECMF with $\beta=2.4$ (i.e. Salpeter), and $\delta t \approx 10 \mathrm{Myr}$, independently of the SFR. The remarkable finding here is that the time-scale of forming a star-cluster population is again found to be about $10 \mathrm{Myr}$.

It therefore appears that galaxies form star-cluster populations such that on a timescale of about $\delta t=10 \mathrm{Myr}$ a statistically complete representation of the ECMF is given. This corresponds to the duty-cycle of molecular clouds (formation from the ISM to emerging star clusters). This constitutes an important insight, but must be challenged by further research, as one possible criticism is that the WKL result may be affected by the choice of ages of the star clusters in the sample (see also Bastian 2008).

\section{The galaxy-wide IMF and new insights on galaxy evolution}

Essentially all of today's understanding of how galaxies evolve and appear rests on the assumption that the stellar initial mass function (IMF) is universal, being roughly a Salpeter-power-law (with index $\alpha=2.35$, Salpeter 1955) perhaps with a flattening below $1 M_{\odot}$. The "canonical $I M F$ " has $\alpha_{1}=1.3$ for $0.08-0.5 M_{\odot}$ and $\alpha_{2}=2.3$ for $0.5<m / M_{\odot}<1$ and $\alpha_{3}=\alpha_{2}$ for $m>1 M_{\odot}$, while the Scalo-field IMF has $\alpha_{3} \approx 2.7$ (Scalo 1986).

With this assumption, the mass-metallicity relation of galaxies needs outflows to carry away metals from dwarf galaxies as otherwise the bend-down of the metal-abundances towards lower-mass galaxies cannot be understood (Kobayashi, Springel \& White 2007). At the same time, the outflows must not remove the gas from late-type dwarf galaxies as these are gas-rich today, with gas-to-stellar mass ratio near 0.8 (as opposed to MW-type disk galaxies where it is about 0.2 ). This poses a problem for the outflow scenario. Also, the galaxy-wide SFR is commonly calculated from a measured $\mathrm{H} \alpha$ luminosity, and the widely-used $\operatorname{SFR}\left(L_{\mathrm{H} \alpha}\right)$ relation (Kennicutt et al. 1994) is linear because the number of ionising massive stars scales linearly with the number of stars formed if the IMF is taken to be invariant.

Based on the neutral-gas masses measured by $21 \mathrm{~cm}$ observations and the SFRs measured with the $\mathrm{H} \alpha$ flux, very low star formation "efficiencies" (i.e. extremely long gasconsumption time-scales of many Hubble times) are deduced for dwarf galaxies, while MW-type galaxies have relatively high efficiencies, i.e. short gas-depletion time-scales of about 3 Gyr.

Only recently has it been fully realised that the assumption of an invariant IMF for galaxies needs revision (Kroupa \& Weidner 2003): The IMF for a galaxy is given, mathematically, by the summation of all IMFs in all forming star clusters. In each star cluster the IMF is the same invariant parent distribution (the above canonical form), except that the stellar masses are bounded above by the available mass in the pre-cluster cloud core. This is a rather elementary physical constraint: for example, star-forming cloud cores of a few $M_{\odot}$ as in Taurus-Auriga-like stellar clusters containing a dozen stars, cannot form stars that weigh more than a few $M_{\odot}$. This issue that the summed IMF of many star clusters cannot be the same as the IMF had already been concluded correctly by Vanbeveren(1982).

\subsection{The $m_{\max }-M_{\mathrm{ecl}}$ relation}

The existence or non-existence of a physical maximum stellar-mass - star-cluster-mass, $m_{\max }-M_{\mathrm{ecl}}$, relation (Weidner \& Kroupa 2006 and references therein), is of much 
importance for the IGIMF theory described below, and can be understood in terms of feedback termination of star-formation in a cloud core through the radiation and winds of the most massive stars together with a time-sequence of stellar-mass buildup such that low-mass stars form, on average, before the massive stars are able to destroy the cloud core.

Since this relation is rather fundamental, not only for our understanding of how a typical star-cluster is assembled, but also for the development of the IGIMF theory, it is worth-while to spend a few words on the $m_{\max }-M_{\text {ecl }}$ relation: A distribution of stars always arises within a cloud core, given that the cores are turbulent and thus have a distribution of density maxima (Padoan \& Nordlund 2002, Li, Klessen \& Mac Low 2003). The stars formed add up to the stellar mass in the core, $\Sigma_{\text {stars }}=M_{\text {ecl }}$. And so the true maximum stellar mass, $m_{\max }$, that can form within a cluster is limited even more strictly than $m_{\max } \leqslant M_{\mathrm{ecl}}$, because $m_{\max }=M_{\mathrm{ecl}}$ would be a cluster consisting of one star leaving no room for the range of stellar masses resulting from turbulent gas dynamics. In other words, isolated O-star formation cannot occur.

However, Maschberger \& Clarke (2008) used observational $m_{\max }-M_{\mathrm{ecl}}$ data to suggest that the existence of a physical $m_{\max }-M_{\mathrm{ecl}}$ relation cannot be confirmed empirically. But, they also note that the random IMF sampling model, which admits isolated O stars (Parker \& Goodwin 2007), is inconsistent with the data. Importantly, the data as used by Maschberger \& Clarke (2008) cannot be interpreted in terms of a currently existing theoretical model. They can, however, be understood rather straightforwardly (Oh et al., in preparation) if the $m_{\mathrm{max}}-M_{\mathrm{ecl}}$ relation exists and (1) if OB stars are shot out from binary-rich and mass-segregated clusters appearing as isolated $\mathrm{O}$ stars and (2) through the rapid dissolution of intermediate-mass clusters through explosive gas-expulsion. This latter process leads to the most massive stars being surrounded by the feeble remnant of the once existing embedded cluster, and thus this $m_{\max }-M_{\text {ecl }}$ datum would be an outlier at too low a value of observed $M_{\mathrm{ecl}}$. Since virtually all those data that do deviate from the relation are indeed outliers at too small $M_{\text {ecl }}$ values, this explanation would appear very natural.

With this in view, the data that do not lie along the proposed $m_{\max }-M_{\text {ecl }}$ relation which are, however, used by Maschberger \& Clarke, have been removed by Weidner \& Kroupa (2006) because they are typically older objects. Instead, Weidner et al. (2007) used these data to estimate the damage to star clusters done by the removal of residual gas in order to constrain the star-formation efficiency in the birth clusters under the assumption that these data were originally on the relation. This demonstrates the difficulty in interpreting the existing data (Maschberger \& Clarke and Parker \& Goodwin vs Weidner \& Kroupa).

In essence, the argument is concerned with the question whether a physical $m_{\mathrm{max}}-M_{\mathrm{ecl}}$ relation exists, or whether star-formation in a star cluster is a mere statistical affair such that stellar masses appear stochastically without any physical boundary conditions. The existence of a pronounced $m_{\max }-M_{\text {ecl }}$ relation for star clusters would imply a selfregulatory behaviour of star-formation on cluster-forming molecular-cloud-core scales. The outline of this physical process would be that as the cloud core begins to contract the physical conditions within it are probably similar to what we observe in TaurusAuriga. As the core contracts the density increases such that ever more massive protostars can condense until their feedback energy suffices to overcome gravitational collapse and the process halts, leaving an exposed and probably largely unbound stellar cluster. This would be a deterministic process in the sense that the initially available gas mass within a given region, i.e. the pressure, would determine the type of star cluster and the mass of its most massive stars, whereby the IMF remains close to the canonical value (as 
determined by observations). Theoretical considerations based on this line of thought do indeed yield a well-pronounced $m_{\max }-M_{\text {ecl }}$ relation (Weidner et al. 2008).

A quantitative counter-argument against the non-existence of a physical $m_{\mathrm{max}}-M_{\mathrm{ecl}}$ relation is, finally, as follows: First of all, the observational evidence is such that in all cases of well-resolved clusters, the IMF is always found to be consistent with the canonical IMF. Now, assuming a purely random-sampling model such that stellar masses can be generated from the canonical IMF and $\Sigma_{\text {stars }}=M_{\text {ecl }}$, where $M_{\text {ecl }}$ is a pre-defined stellar cluster mass, there are occurrences such that $m_{\mathrm{max}} \approx M_{\mathrm{ecl}}$ (Parker \& Goodwin 2007). These would be the observed 4 per cent isolated O stars. However, there would also be cases where the first cluster star picked from the IMF is massive enough to destroy the cloud core through feedback energy such that no further stars can form. The resulting number of isolated $\mathrm{O}$ stars would therefore outnumber the observed number of isolated O stars.

Given the above and the existence of known physical processes that can explain the existence of apparently isolated $O$ stars as being either ejected stars $(\S 2)$ or remnants of intermediate-mass clusters that rapidly dissolved after residual gas expulsion (Weidner et al. 2007), it follows that the purely random sampling model for creating star clusters ("the non-existence model") probably needs to be rejected.

\subsection{The IGIMF}

By taking into account the existence of a physical $m_{\max }-M_{\text {ecl }}$ relation, i.e. that a cluster of stellar mass $M_{\text {ecl }}$ cannot have stars with $m \geqslant m_{\max }=\mathrm{fn}\left(M_{\mathrm{ecl}}\right)$, and then adding up all the so-constructed IMFs for a co-eval embedded star-cluster population that is a power-law with Salpeter index $\beta=2.35$ (see also $\S 1$ and $\S 5$ ), it follows that the resulting "integrated galactic IMF" (the IGIMF) is steeper above about $1.3 M_{\odot}$ than the canonical IMF (Vanbeveren 1982, Kroupa \& Weidner 2003). This immediately solves the finding that the field-star IMF is steeper than the IMF in individual clusters (Vanbeveren 1983, Vanbeveren 1984). Thus, the Scalo-field-IMF, which has $\alpha_{3} \approx 3$, is unified with the canonical IMF $\left(\alpha_{3}=2.3\right)$ in a straight-forward way.

Also, since the maximum cluster mass, $M_{\text {ecl,max }}$, that can form within the time span $\delta t$ within a galaxy depends on the SFR of the galaxy ( $\S 5$ above), the IGIMF becomes SFR dependent, such that galaxies with a low SFR have steeper IGIMFs because of the $m_{\max }-M_{\text {ecl }}$ relation (Weidner \& Kroupa 2006, Pflamm-Altenburg et al. 2007). More importantly, the maximum stellar mass, $m_{\mathrm{m}}$, forming in a galaxy decreases with decreasing SFR.

For the MW, which has a SFR of a few $M_{\odot} / \mathrm{yr}$, the IGIMF turns out to have an index $\alpha_{\text {IGIMF }} \approx 3$ above a stellar mass of $1.3 M_{\odot}$ and $m_{\mathrm{m}}=150 M_{\odot}$, while for a galaxy with a SFR near $10^{-3} M_{\odot} / \mathrm{yr}, \alpha_{\mathrm{IGIMF}} \approx 3.3$ and $m_{\mathrm{m}} \approx 20 M_{\odot}$. Such a variation of the IGIMF index has been reported by Hoversten \& Glazebrook(2008) on the basis of analysing a hundred-thousand star-forming galaxies in the SDSS survey. Of relevance here is that the gamma-ray flux from decaying ${ }^{26} \mathrm{Al}$ yields a current SFR of about $4 M_{\odot} / \mathrm{yr}$ for the MW if the (Scalo) IGIMF slope, $\alpha_{3}=2.7 \approx 3$, for the Milky Way field is used (Diehl et al. 2006). This constitutes an independent confirmation of the SFR- $\alpha_{\text {IGIMF }}$ relation for the case of the MW. But further confirmation, for example by measuring the IGIMF in dwarf galaxies, would be essential to test this theory.

\subsection{The mass-metallicity relation of galaxies}

The IGIMF theory described above immediately explains the mass-metallicity relation of galaxies without the need of additional physical processes (Köppen et al. 2007). This is so because low-mass galaxies have a deficit of massive stars per low-mass star when 
compared to more massive galaxies - they have top-light IGIMFs. It follows that galaxywide metal production is compromised increasingly with decreasing galaxy mass.

This does not mean that outflows and infall do not occur, but that these processes probably play a secondary role in establishing the metal content of galaxies.

Noteworthy is that in the currently established picture outflows need to be invoked to remove the metals but such that the gas is not blown out, given that late-type dwarfs are metal-poor and very gas rich. In the IGIMF theory the metals are not produced in the first place and so unwanted gas blow-out is not an issue.

\subsection{Gas consumption time scales}

The IGIMF theory also implies that the $\mathrm{H} \alpha$-luminosity-SFR calibration in general use needs to be re-calibrated. This generally used relation leads to the widely accepted result that dwarf galaxies have very low star-formation "efficiencies", i.e. gas-consumption timescales longer than many Hubble times. Pflamm-Altenburg et al. (2007) have re-calibrated the $\mathrm{H} \alpha$-luminosity-SFR relation based on the above IGIMF theory. Here, a dwarf galaxy with a low SFR is producing significantly fewer ionising photons due to the top-light IGIMF than a massive galaxy. For a given measured $\mathrm{H} \alpha$ luminosity the true SFR would therefore be significantly higher than hitherto thought.

Without any parameter adjustments, the immediate result is that the true SFRs of dwarf galaxies are orders of magnitude higher than thought until now, implying neutralgas-consumption time-scales of about 3 Gyr for all galaxies that contain neutral gas, and that the SFR is strictly proportional to the neutral gas mass of a galaxy, $S F R=$ $1 /(3 \mathrm{Gyr}) M_{\text {gas }}$. Naturally, this leads to a very major revision of our understanding of galaxy evolution and of the galaxy-wide star-formation process. Note that near $M_{\text {gas }}=$ $10^{9.5} M_{\odot}$ the Kennicutt et al. (1994) finding, that the time-scale for gas consumption is about $3 \mathrm{Gyr}$, remains valid.

The star-formation efficiency (the fraction of gas that turns into stars) over a time-scale of $\delta t=10 \mathrm{Myr}$ becomes $\epsilon=(1 / 300) M_{\text {gas }}$, i.e. every $10 \mathrm{Myr}$ a (not strongly interacting) galaxy turns 0.3 per cent of its neutral gas mass into stellar mass. According to the IGIMF theory, this would be true for all not strongly interacting galaxies with neutral gas.

\subsection{The radial $\mathrm{H \alpha}$ star-formation cutoff}

The IGIMF theory also immediately and naturally explains the existence of an $\mathrm{H} \alpha$ cutoff radius in disk galaxies. The current understanding, based on applying a universal IMF to galaxies, is that the radial $\mathrm{H} \alpha$-emission cutoff comes about because beyond the $\mathrm{H} \alpha$-cutoff radius star formation is suppressed due to dynamical processes. Basically, star-forming cloud cores cannot assemble beyond a particular radius. However, using recent GALEX observations (Boissier et al. 2007) show UV emission from young stars to continue beyond this cutoff radius, in contradiction to the theoretical work.

A local IGIMF description can be formulated rather straightforwardly by connecting the local star-formation rate surface density with the local neutral-gas surface density in a disk galaxy. With this star-formation rate surface density, the local star cluster density can be computed, and by summing them all up, a local IGIMF (LIGIMF) follows. Integration of the LIGIMF over radial annuli then yields the $\mathrm{H} \alpha$ flux density in dependence of the radius, and it follows quite trivially that it decays much more rapidly than the starformation rate density (Pflamm-Altenburg \& Kroupa 2008). An interesting outcome of this work is that the star-formation rate surface density is linearly proportional to the gas surface density, $\Sigma_{\mathrm{SFR}} \approx 1 /(3 \mathrm{Gyr}) \Sigma_{\text {gas }}^{N}$, with $N=1$. 


\subsection{Summary: IGIMF}

The implications of all of this work would be that a paradigm shift in galaxy evolution may be emerging such that the star-formation rate (density) is proportional to the neutral gas mass (density). Dwarf galaxies differ from large disk galaxies only in terms of the level of star formation because the latter have a larger neutral gas mass which supports more star-formation activity. Further implications of this are being looked into now.

\section{Conclusions}

The above shows that we have been missing important ingredients in our understanding of the astrophysics of disk galaxies if the physics of star clusters as the fundamental galactic building blocks is omitted. As important examples the following have been noted:

- Young cluster cores are stellar accelerators dispersing massive stars over large distances from their birth sites.

- The binary fraction and SN Ia rate are defined by the star-cluster population a galaxy has been able to generate over its life time.

- Galactic disks may thicken if they form ensembles of "popping" clusters.

- Star-clusters in a statistically complete ensemble, such that they represent the embedded cluster mass function, appear to form on a time-scale of about $10 \mathrm{Myr}$.

- At the same time, by realising that we must see galaxies as made up of many (mostly dissolved) star clusters we are readily led to find a new understanding of the mass-metallicity relation of galaxies, of the radial $\mathrm{H} \alpha$ cutoff and therewith the relation of neutral gas content to the level of star formation.

- Following on from this, it emerges that dwarf irregular galaxies consume their neutral gas content on the same time scale (about $3 \mathrm{Gyr}$ ) as major disk galaxies, and that it is only the mass of neutral gas that drives the macroscopic evolution of these systems in terms of the buildup of stellar mass and metals.

- The last two points are a result of correctly counting massive stars in galaxies in dependence of their SFRs: massive disk galaxies have a higher SFR than low-mass irregular galaxies and consequently their IGIMF is flatter and the upper mass limit of forming stars is higher. The implications of this suggest a possibly major paradigm shift in our knowledge of how galaxies evolve.

\section{Acknowledgments}

I would like to thank the organisers for the invitation to present this material at a most memorable meeting in Copenhagen, and Jan Pflamm-Altenburg and Carsten Weidner for very important contributions. This contribution I wrote in Vienna and Canberra, and would like to thank the respective hosts (Gerhardt Hensler, Christian Theis and Helmut Jerjen) for their kind hospitality.

\section{References}

Bastian, N. 2008, MNRAS, in press (astro-ph:0807.4687)

Bastian, N., Emsellem, E., Kissler-Patig, M., \& Maraston, C. 2006, A\& A, 445, 471

Baumgardt, H. \& Kroupa, P. 2007, MNRAS, 380, 1589

Baumgardt, H., de Marchi, G., \& Kroupa, P. 2008, ApJ, in press, astro-ph/0806.0622

Baumgardt, H., Kroupa, P., \& Parmentier, G. 2008, MNRAS, 384, 1231

Boissier, S., et al. 2007, ApJS, 173, 524

Clarke, C. J., \& Pringle, J.E. 1992, MNRAS, 255, 423

Davies, M. B., et al. 2006, New Astronomy, 12, 201 
de La Fuente Marcos, R. 1997, A\&\&A, 322, 764

Diehl, R., et al. 2006, Nature, 439, 45

Duchêne, G. 1999, A\&̈A, 341, 547

Egusa, F., Sofue, Y., \& Nakanishi, H. 2004, PASJ, 56, L45

Elmegreen, B. G. \& Elmegreen, D. M. 2006, ApJ, 650, 644

Fellhauer, M. \& Kroupa, P. 2005, ApJ, 630, 879

Giersz, M. \& Spurzem, R. 2003, MNRAS, 343, 781

Goodwin, S. P. \& Kroupa P. 2005, AछA, 439, 565

Goodwin, S. P., Kroupa, P., Goodman, A., \& Burkert A. 2007, Protostars and Planets V, 133

Gvaramadze, V. V. \& Bomans, D. J. 2008, A\&A, in press, astro-ph/0809.0650

Heggie, D. C., Trenti, M., \& Hut P. 2006, MNRAS, 368, 677

Hoversten, E. A. \& Glazebrook, K. 2008, ApJ, 675, 163

Ivanova, N., Belczynski, K., Fregeau, J. M., \& Rasio, F. A. 2005, MNRAS, 358, 572

Kennicutt, R. C. Jr., Tamblyn, P., \& Congdon, C. E. 1994, ApJ, 435, 22

Kobayashi, C., Springel, V., \& White, S. D. M. 2007, MNRAS, 376, 1465

Köppen, J., Weidner, C., \& Kroupa, P. 2007, MNRAS, 375, 673

Kouwenhoven, M. B. N., Brown, A. G. A., Portegies, Zwart S. F., \& Kaper, L. 2007, A\& A, 474, 77

Kroupa, P. 1998, MNRAS, 298, 231

Kroupa, P. 2002, MNRAS, 330, 707

Kroupa, P. 2008, S. Aarseth, Ch. Tout, R. Mardling (eds.), The Cambridge N-body Lectures (Lecture Notes in Physics Series, Springer Verlag), astro-ph/0803.1833

Kroupa, P., \& Boily, C. M. 2002, MNRAS, 336, 1188

Kroupa, P. \& Weidner, C. 2003, ApJ, 598, 1076

Kroupa, P., Aarseth, S., \& Hurley, J. 2001, MNRAS, 321, 699

Kroupa, P., Petr, M. G., \& McCaughrean, M. J. 1999, New Astronomy, 4, 495

Küpper, A. H. W., Kroupa, P., \& Baumgardt, H. 2008, MNRAS, 389, 889

Lada, C. J. \& Lada, E. A. 2003, Annual Review of A\&A, 41, 57

Lada, C. J., Margulis, M., \& Dearborn, D. 1984, ApJ, 285, 141

Larsen, S. S. 2002a, AJ, 124, 1393

Larsen, S. S. 2002b, in: D. Geisler, E. K. Grebel, \& D. Minniti (eds.), Extragalactic Star Clusters (San Francisco: Astronomical Society of the Pacific, 2002., IAUS 207), p. 421

Larsen, S. S. 2004a, $A \mathscr{E} A, 416,537$

Larsen, S. S. 2004b in: H. J. G. L. M. Lamers, L. J. Smith \& A. Nota (eds.), The Formation and Evolution of Massive Young Star Clusters (San Francisco: Astronomical Society of the Pacific, 2004., IAUS 322,), p.19

Li Y., Klessen, R. S., \& Mac Low, M.-M. 2003, ApJ, 592, 975

Marks, M., Kroupa, P., \& Baumgardt, H. 2008, MNRAS, 386, 2047

Maschberger, T. \& Clarke, C. J. 2008, (astro-ph/:0808.4089)

Meylan, G. \& Heggie, D. C. 1997, The Astronomy and Astrophysics Review, 8, 1

Padoan, P. \& Nordlund, A. 2002, ApJ, 576, 870

Parker, R. J. \& Goodwin, S. P. 2007, MNRAS, 380, 1271

Parmentier, G., Goodwin, S. P., Kroupa, P., \& Baumgard, H. 2008, ApJ, 678, 347

Pflamm-Altenburg, J. \& Kroupa, P. 2006, MNRAS, 373, 295

Pflamm-Altenburg, J. \& Kroupa, P. 2008, Nature, in press

Pflamm-Altenburg, J., Weidner, C., \& Kroupa, P. 2007, ApJ, 671, 1550

Portegies Zwart, S. F., McMillan, S. L. W., \& Makino, J. 2007, MNRAS, 374, 95

Renaud, F., Boily, C. M., Fleck, J., Naab, T., \& Theis, C. 2008, MNRAS Letters. in press, astro-ph/0809.2927

Salpeter, E. E. 1955, ApJ, 121, 161

Scalo, J. M. 1986, Fundamentals of Cosmic Physics, 11, 1

Schilbach, E., Roeser, S. 2008, A\& A, 489, 105

Shara, M. M., \& Hurley, J. R. 2002, ApJ, 571, 830

Trenti, M., Heggie, D. C., \& Hut, P. 2007, MNRAS, 374, 344

Vanbeveren, D. 1982, A\&A, 115, 65 
Vanbeveren, D. 1983, A\& A, 124, 71

Vanbeveren, D. 1984, A\&BA, 139, 545

Weidner, C., \& Kroupa, P. 2005, ApJ, 625, 754

Weidner, C. \& Kroupa, P. 2006, MNRAS, 365, 1333

Weidner, C., Kroupa, P., \& Larsen, S. S. 2004, MNRAS, 350, 1503

Weidner, C., Kroupa, P., \& Goodwin, S. P. 2008, in preparation

Weidner, C., Kroupa, P., Nürnberger, D. E. A., \& Sterzik, M. F. 2007, MNRAS, 376, 1879

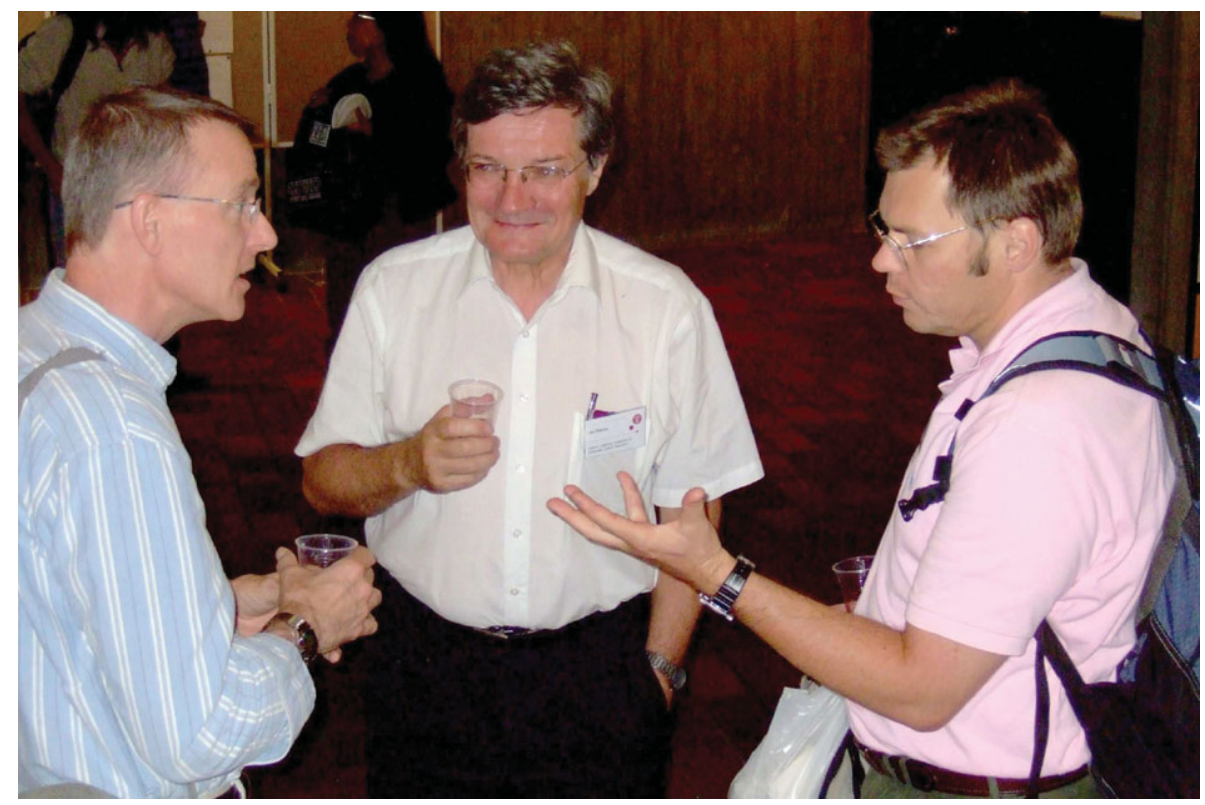

Bruce Elmegreen, Jan Palouš, and Pavel Kroupa continuing the discussion after Pavel's talk.

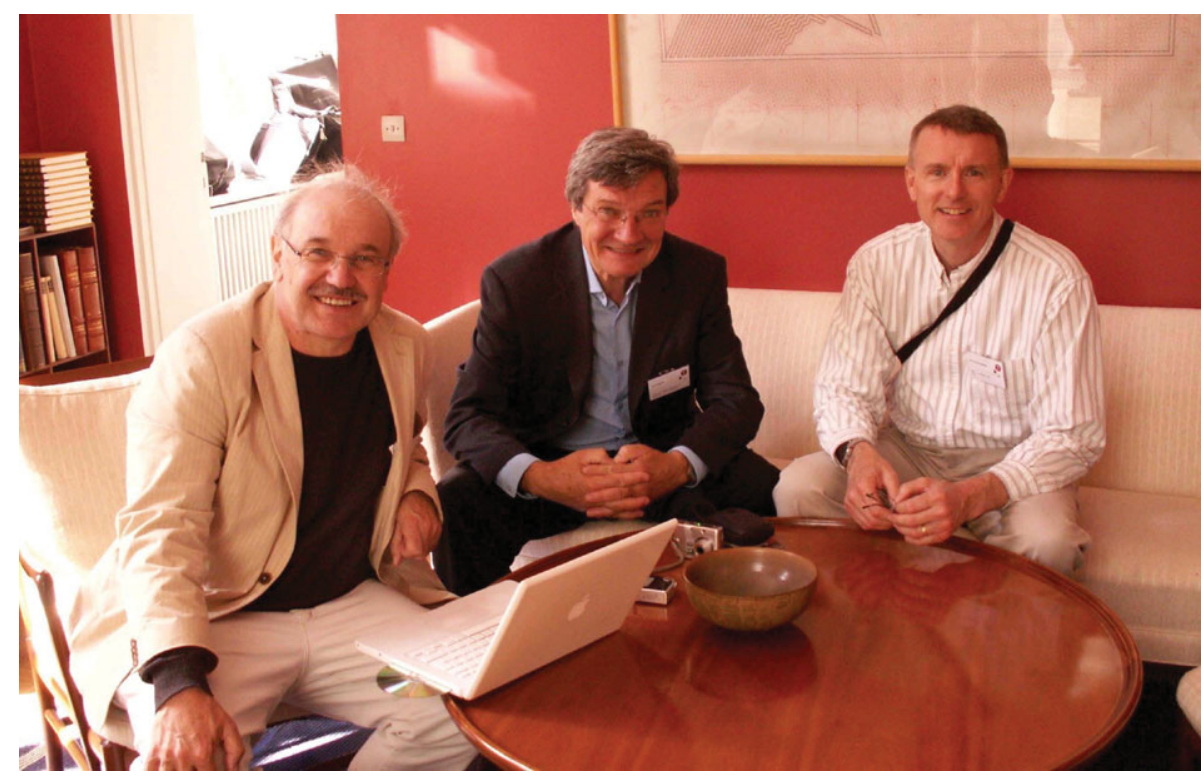

Hans Zinnecker, Jan Palouš and Bruce Elmegreen at Carlsberg (photo: Bruce Elmegreen). 